\title{
Flow Injection Analysis of Iron in Presence of Uranium
}

\author{
S Chandramouleeswaran ${ }^{1}$ and Jayshree Ramkumar ${ }^{1^{*}}$ \\ ${ }^{1}$ Analytical Chemistry Division, Bhabha Atomic Research Centre, Mumbai-85, India
}

Received: 02 June, 2017; Accepted: 13 July, 2017; Published: 24 July, 2017

*Corresponding author: Jayshree Ramkumar, Analytical Chemistry Division, Bhabha Atomic Research Centre, Mumbai-85, India, Tel: 91-022-25592224;

E-mail: jrk@barc.gov.in

\begin{abstract}
The determination of trace amounts iron is imperative in various fields including nuclear fuel characterization. Despite various reports on developed methodologies, there exists an ever increasing interest to develop newer methods which are rapid and simple. In this paper, single flow injection analysis of iron using flow injection analyzer with UV Visible detector has been discussed. Since the method is dependent on formation of a coloured species between iron and a chromophoric reagent, it was necessary to optimize the chromophore in addition to other experimental parameters. Different reagents like orthophenanthroline, 8 Hydroxy Quinoline and xylenol orange (XO) were evaluated and $\mathrm{XO}$ was found to be most suitable in presence of uranium. The optimized concentration of XO and acidity were $0.005 \%$ and $0.05 \mathrm{M}$ respectively. A calibration was obtained for a concentration range of 10-80 ng of iron with a R2 of 0.9934. The calibration of iron in presence of varying concentrations of uranium showed that up to $1 \mathrm{mg} / \mathrm{mL}$ of uranium, the linearity of calibration and sensitivity of detection is unaffected. However the sensitivity was severely affected at higher uranium concentrations. The limit of detection was $1 \mathrm{ng}$ of Fe and the precision was found to be $1.8 \%$.
\end{abstract}

Keywords: Single flowinjection analyzer; Iron;Spectrophotmetry; Chromophore; Mixture

\section{Introduction}

Analytical chemistry involves the science of providing information about the target in order to facilitate diagnoses and the making of educated, effective decisions. Trace level determination of metallic impunity is one of the imperative steps of chemical quality control of nuclear fuels. The spectra of $U$ are so complex that a direct determination of the metal ions in presence of $U$ becomes very difficult to interpret. Therefore separation becomes essential and regardless of the substantial furtherance in sensitivity using new instrumentation, conventional separation techniques are recurrently used to surmount interferences from matrix elements as well as to improve detection limits $[1,2]$. However separation results in increase of time of analysis and also great care is needed as there is a greater chance of contamination during reaction process. ICP-AES based method for direct determination of trace metallic constituents without any separation has been reported [3]. Another technique which is very useful is the Flow Injection Analysis (FIA) which has several advantages like small sample size, high sample throughput due to shorter reaction times, reproducibility, reliability, and ease of automation [4]. The concept of FIA depends on a combination of three factors: reproducible sample injection volumes, controllable sample dispersion, and reproducible timing of the injected sample through the flow system. The common feature of all Flow Injection (FI) methods is that the characteristics of fluid flow in closed medium are exploited for one or more of the following operations on a controlled amount of sample (a) the transport of samples, (b) the chemical pretreatment of samples and (c) the presentation of samples to a chemical instrument. As all samples and standards have identical residence times, the kinetic limitations of conventional analytical methods, in which samples and standards arc handled in parallel, do not apply. Thus it is not necessary for any of the chemical or physical processes in the system to be at equilibrium and restrictions on the stability of both the product and the reagents can be relaxed. Single Line Manifold (SLM) in which a reagent carrier stream continuously flows and a fixed volume of sample is injected into the flowing reagent carrier results in dispersion due to the combination of laminar flow (in which a parabolic velocity profile develops between the center stream line and the wall) and diffusion. Flow injection analysis of iron in various matrices has been reported [5-12].

In this paper, a simple direct single line flow injection methodology without any pre concentration for determination of iron in its binary mixture containing uranium has been developed. Different experimental parameters that affect the sensitivity are optimized.

\section{Experimental}

\section{Reagents}

Stock solutions of metal ions were prepared using their respective salts in dilute water. The stock solution of iron was 1 $\mathrm{mg} / \mathrm{mL}$. Orthophenanthroline, 8 Hydroxy Quinoline and xylenol orange were obtained from E.Merck, Germany. Solutions of sodium acetate buffer, 1,10-phenanthroline solution (1 g/L), and hydroxylamine hydrochloride solution $(100 \mathrm{~g} / \mathrm{L})$ were prepared using appropriate salts. 


\section{Instrumentation}

The simplest flow injection analyzer shown in Figure 1 consists of a pump, for pushing the carrier (appropriate chromophoric reagent) through a narrow tube; an injection port, through which a well-defined volume $(20 \mathrm{uL})$ of a sample solution (iron or its mixture) is injected into the carrier stream in a reproducible manner; and a reaction coil which facilitates the reaction between sample and the chromophoric reagent in carrier to form a coloured species that can be detected in a UV-Vis flow detector at a fixed appropriate wavelength and the output is recorded. The flow injection analysis was carried out using JASCO UV 2070 Plus flow detector. The calibration curve was obtained by the measurement of peak area. The various experimental parameters including the chromophoric reagent were varied to get a good method for determination of iron.

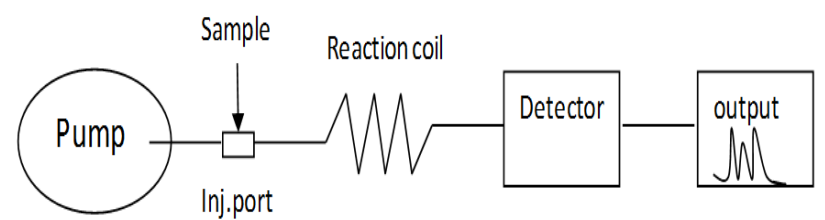

Figure 1: Schematic Representation of single flow injection analyzer

\section{Results and Discussion}

Analysis of trace level ions is of great interest and there is always an ongoing research in this field. Several techniques including spectrochemical methods have been employed for the determination of iron. The advances in excitation sources such as Inductively Coupled Plasma (ICP) have considerably enhanced the sensitivity, dynamic range and precision of these methods. However, in most of the instances, the direct determination of ions of interest is not possible due to possible spectral interferences. Uranium has a very complex spectrum with thousands of emission lines spread over the entire range of 200-500 $\mathrm{nm}$. These lines severely interfere with the analytical lines of the elements ( Fe) of interest. Hence it is desirable to separate the major matrix for eliminating the interferences. The different aspects of separation of matrix prior to analysis of element of interest viz iron by ICP AES have been discussed in our earlier study [1].

In the present study, it was of great interest to see whether the matrix separation could be avoided and a simple direct methodology could be evolved for the determination of iron in presence of large amount of uranium. Hence Flow injection analysis seemed as a good alternative. For this, the simplest form of FIA known as single line FIA with no separation was adopted (Figure 1). The sample is injected into a continuous flow of reagent solution (carrier), dispersed, and transported to detector and sample dispersion is controlled through the suitable choice of the injected sample volume, flow rate of carrier, length of the reaction coil, and diameter of the tubing used. The analytical procedure is based on the reaction of $\mathrm{Fe} 3+$ with chromophoric reagent (CA), in the absence and presence of matrix uranium ion.
Therefore the method developed was based on optimization of chromophore, solution conditions, flow conditions etc.

\section{Optimization of flow rate of reagent solution}

The first important step in method development was the flow optimization. The main advantage of this single line FIA is that there is no back pressure due to the column. The reagent flow rate should not be too low as it results in very broad peaks. Very high flow conditions are not quite advantageous in a single line FIA as it does not ensure complete chemical reaction between the chromophore and solute of interest. The effect of the flow-rate of the peristaltic pump was examined by varying the range from 1- 5 $\mathrm{mL} / \mathrm{min}$. The optimized flow rate was maintained at $1.5 \mathrm{~mL} / \mathrm{min}$ in all subsequent experiments.

\section{Choice of chromophoric reagents}

Since the method is dependent on formation of a coloured species between iron and a chromophoric reagent, it was necessary to optimize the chromophore. Different reagents were evaluated for their performance as chromophores. Orthophenanthroline was tried initially. It is well known that orthophenanthroline is used for the determination of iron in its +2 state [13]. However, in the present study, iron being in its +3 state had to be reduced prior to its determination with orthophenanthroline. The reduction was carried out using hydroxyl amine hydrochloride. The carrier contained a mixture of orthophenanthroline (1 g/L) and hydroxylamine hydrochloride $(100 \mathrm{~g} / \mathrm{L})$. The measurements were carried out at $515 \mathrm{~nm}$. A linear calibration plot of peak area vs concentration of iron in the range $10-80 \mathrm{ng}$ with a R2 of 0.985 was obtained. The main aim of the study is to develop a methodology for determination of iron in presence of uranium. It was seen that even in presence of only 3 times higher uranium concentration, the linear variation using orthophenanthroline was lost. Therefore this procedure was not suitable and an alternative chromophoric reagent had to be thought of 8 hydroxy quinoline is known to be a chromogenic reagent for extractive spectrophotometric determination of iron [14]. Based on the report, it was thought of adopting the sulphonic acid for the direct flow injection methodology. The absorbance values of the green coloured complex measured at $600 \mathrm{~nm}$ were lower than that of orthophenanthroline but a linear calibration was obtained. However, it was seen that in presence of equal concentration of uranium, the absorbance values not only decrease but also lose their linear variation. This could be attributed to the complexation of uranium with oxine. Thus the need for a superior chromophore arises. Xylenol Orange (XO) has long been known as a good chelating reagent and quantitative indicator of a wide range of cat ions, including iron in its tervalent state $[15,16]$. For determination purposes, $\mathrm{XO}$ has to be in much excess of the iron concentration. An additional advantage of xylenol orange is that uranyl ion forms very weakly coloured chelate with it [17]. Based on these advantages, it was thought of using $\mathrm{XO}$ as a chromophore for single line FIA determination of $\mathrm{Fe} 3+$ in presence of uranium. The measurements using $\mathrm{XO}$ as chromophore for determination of Fe3+ were carried out at 574 nm. Blank measurements were also carried out. 


\section{Effect of Acidity of the solution}

The acidity is a very important parameter as it prevents the hydrolysis of iron and also reduces the contributions from the blank. The entire determinations were carried out in acidic conditions wherein the blank was orange in colour and did not show any interference in the wavelength of measurement (574 $\mathrm{nm})$.

\section{Effect of Chromophore concentration}

The concentration of the chromophore solution was of great importance. These studies were carried out using $80 \mathrm{ng}$ of Fe in acidic medium and the concentration of XO in the carrier was varied. The results are given in Figure 2.

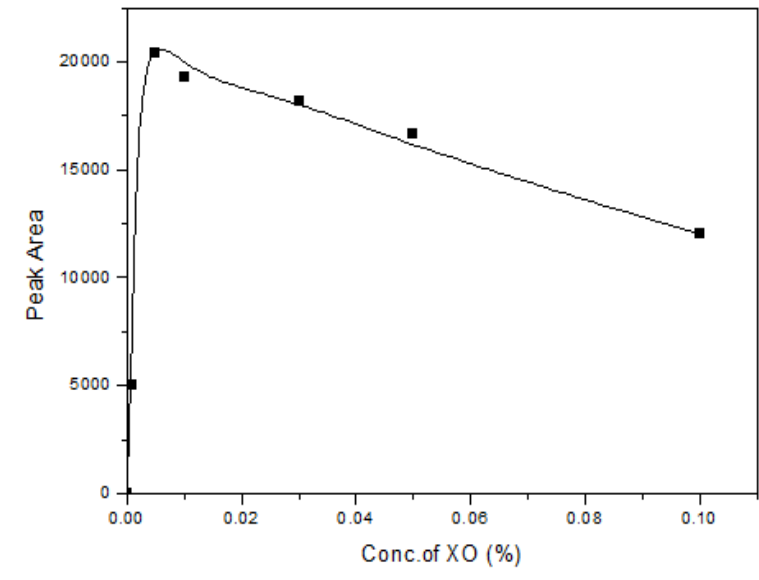

Figure 2: Effect of Chromophore concentration on detector response

The detector response in terms of peak area was given as a function of concentration of chromophore xylenol orange in Figure 3. It was found that the change in concentration gave a change in the detector response. Also it was important to assess whether the linear calibration as a function of iron concentration was unaffected with increase in the concentration of XO. It was seen that at XO concentration of $0.1 \%$, the interference from blank itself was high and no reproducible and stable signal was obtained. Hence the optimum concentration of $0.005 \%$ XO was used for further studies.

\section{Calibration Curve}

Under the optimized conditions, the determination of pure iron was carried out. The trace obtained during analysis is shown in Figure 3a.The calibration obtained for pure iron solution given in Figure 3b shows a good linear fit with a R2 value of 0.9934 which was within the accepted range of the official published guidelines and showed good linear relationship of the method.

Since the main aim of the present study was to carry out the determination of iron in presence of large amounts of uranium, the calibration was studied using binary mixtures containing varying amount of uranium. The results are shown in Figure 4. It is seen that up to $1 \mathrm{mg} / \mathrm{mL}$ of uranium, the calibration curve is unaffected.
But with further increase in the uranium concentration, there is a marked decrease in the detector response and also sensitivity of the method.

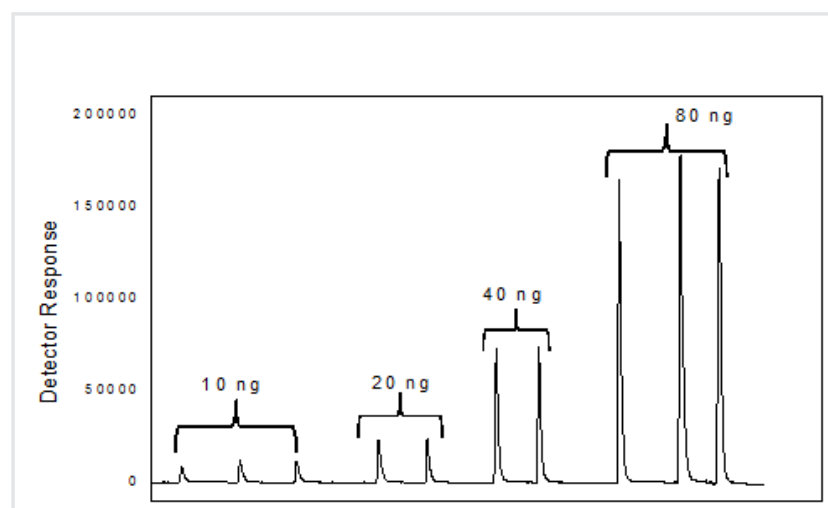

(A)

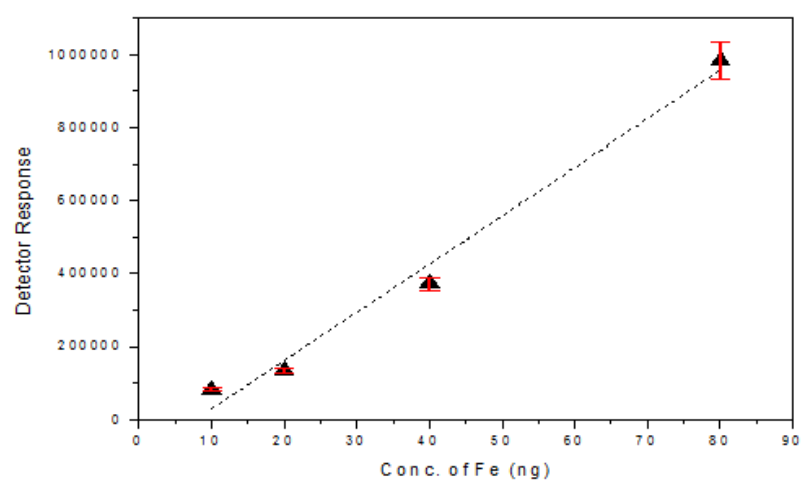

(B)

Figure 3: Studies using pure Fe (A) trace for different amounts and (B) Calibration curve

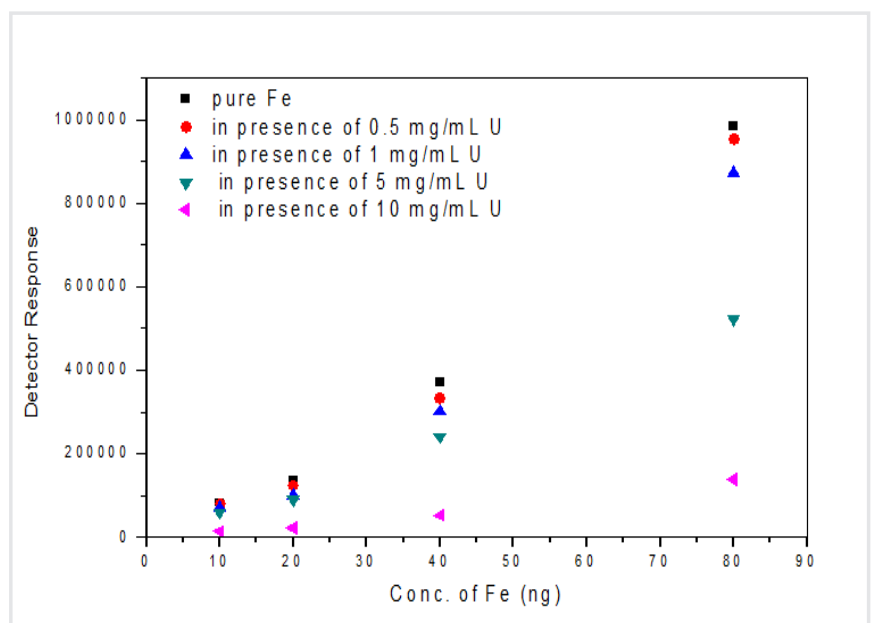

Figure 4: Calibration Curve of Fe in presence of varying concentrations of $U$ 
The main aim of development of this work was to apply to uranium samples. The determination of iron in uranium using TBP extraction involves a large number of steps [18]. A novel filter less preconcentration methodology was developed using a manifold of Flow Injection Analysis (FIA) wherein iron is preconcentrated on the inner walls of a tubular nylon reactor resulting in enrichment factors of 30-35 using optimized conditions and then eluted using $\mathrm{HCl}$ and analyzed by Flame Atomic Absorption Spectrometry (FAAS) [19]. The limit of detection of $1.8 \mathrm{mg} / \mathrm{L}$ was obtained and the sample throughput was quite high with a sampling frequency of 40 per hour and used for analysis of small amounts of iron in drinking water, in digested samples and in saline water. Normally in the samples the maximum permissible amount of $\mathrm{Fe}$ is $150 \mathrm{ppm}$ and most of the samples contain less than $150 \mathrm{ppm}$. Prior to sample analysis about $1 \mathrm{~g}$ of $\mathrm{U}$ is digested and made upto $25 \mathrm{~mL}$ and therefore final concentration of $\mathrm{Fe}$ in solution is about $6 \mathrm{ppm}$. However, it is seen that upto $1 \mathrm{mg} /$ $\mathrm{mL}$ of uranium can be tolerated in this developed methodology. So even if the amount of Uranium matrix is fixed at $1 \mathrm{mg} / \mathrm{mL}$, it is seen that the maximum amount of Fe that will be present will be $3 \mathrm{ng}$ in the final sample loop and this is below the limit of quantification of the developed methodology. Hence to make it applicable to uranium samples containing a maximum of 150 ppm of Fe, the present methodology has to be modified and this is not within the scope of this paper.

However it is worth mentioning that this can be used to give an indication of whether or not the iron in uranium sample exceeds the specified value. Also the developed methodology does not involve any separation procedure.

\section{Statistical treatment of data}

The variation of the slope and $\mathrm{R} 2$ values of the calibration curves obtained for iron in presence of varying concentrations of uranium is given in Figure 5. It is quite clear that at high concentrations of 5 and $10 \mathrm{mg} / \mathrm{mL}$ of uranium, there is a great reduction of sensitivity as seen by the reduction of value of slope. However, even at this level of uranium, the linearity is not affected much as seen by a less degree of change in R2 value.

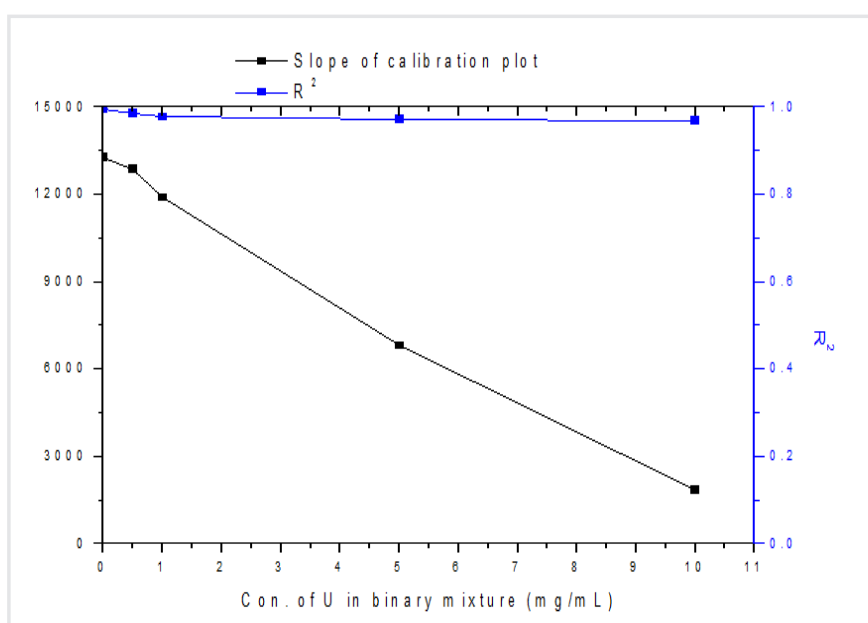

Figure 5: Statistical parameters of Calibration Curve of Fe (varying concentrations of $\mathrm{U}$ )

\section{Validation of the methodology}

The developed procedure was tested for its accuracy precision as given by the inter- and intra-day repeatability respectively by injecting two sets of binary mixtures of Fe and $U$, containing a fixed concentration of uranium at $1 \mathrm{mg} / \mathrm{mL}$. The iron concentrations in the two sets were 10 and $40 \mathrm{ng}$ respectively. The results are given in Figure 6. It is seen that the developed method showed reproducibility over the entire period of study indicating the robustness of the procedure.

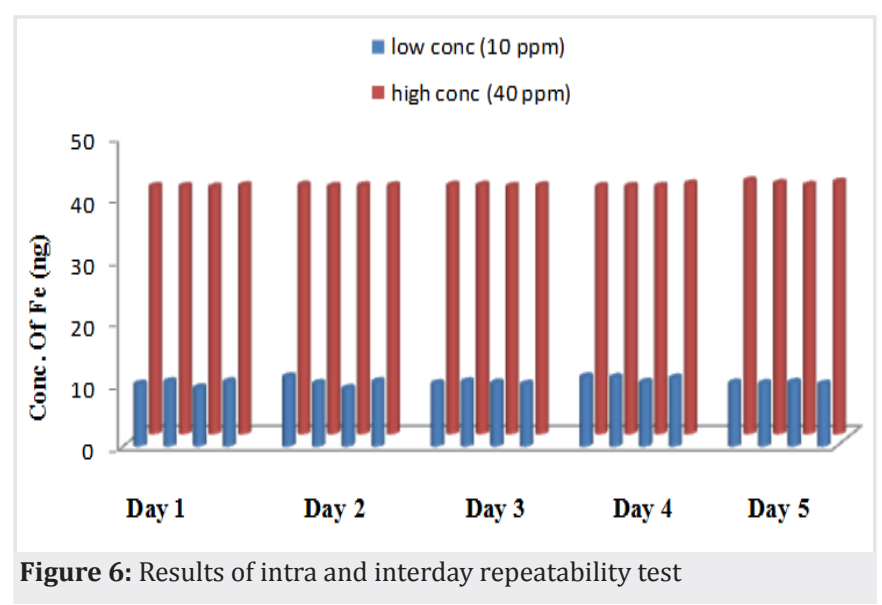

\section{Conclusion}

A single flow injection analysis of iron using flow injection analyzer with UV Visible detector has been discussed. The choice of chromophore was very important as the analysis was needed in presence of large amounts of uranium. Of the various chromophoric reagents evaluated, Xylenol orange (XO) was found to be the most suitable. The optimized concentration of $\mathrm{XO}$ and acidity were $0.005 \%$ and $0.05 \mathrm{M}$ respectively. A calibration was obtained for a concentration range of 10-80 ng of iron with a R2 of 0.9934 . The calibration of iron in presence of varying concentrations of uranium showed that upto $1 \mathrm{mg} / \mathrm{mL}$ of uranium, the sensitivity and linearity was maintained. At higher concentrations of uranium, there was a loss of sensitivity. The limit of detection was $1 \mathrm{ng}$ and the precision was found to be 1.8 $\%$. The ruggedness and robustness of the procedure is understood by the intraday and inter day repeatability respectively.

\section{Acknowledgements}

The authors would like to thank Dr. P. D. Naik, AD Chemistry Group, BARC for his constant support.

\section{References}

1. Gopalakrishnan M, Radhakrishnan K, Dhami PS, Kulkarni VT, Joshi MV, Patwardhan $A B$, et al. Determination of Trace Impurities in Uranium, Thorium and Plutonium Matrices by Solvent Extraction and Inductively Coupled Atomic Emission Spectrometry. Talanta. 1997;44:169-176. doi:10.1016/S0039-9140(96)02027-9

2. Sengupta A, Adya VC. Determination of analytes at trace level in uranium matrix by ICP-AES without chemical/physical separation. J 
Radioanal Nuc Chem. 2014;299:2023-2026.

3. Andac M, Asan A, Isildak I. A simple flow injection spectrophotometric determination method for iron(III) based on 0-acetylsalicylhydroxamic acid complexation. Chem. Pap. 2009;63(3):268-273.

4. Kartikeyan S, Vijayalekshmy B, Chandramouleeswaran S, Rao TP, Iyer CPS. Flow Injection On-Line Preconcentration and Flame AAS Determination of Copper, Cadmium and Lead in Marine Sediment Samples. Anal Lett. 1997;30(5):1037-1050. doi: 10.1080/00032719708002316

5. Mortatti J, Krug FJ, Pessenda LCR, Zagatto EAG, Jorgensen SS Determination of iron in natural waters and plant material with 1,10-phenanthroline by flow injection analysis. Analyst. 1982;107:659-663.

6. Borman CJ, Sullivan BP, Eggleston CM, Colberg PJS. The Use of FlowInjection Analysis with Chemiluminescence Detection of Aqueous Ferrous Iron in Waters Containing High Concentrations of Organic Compounds Sensors. 2009;9(6):4390-4406.

7. Measures CI, Yuan J, Resing JA. Determination of iron in seawater by flow injection analysis using in-line preconcentration and spectrophotometric detection. Marine Chem. 1995;50(1-4):3-12.

8. Blain S, Treguer P. Ironn (II) and iron(III) determination in sea water at the nanomolar level with selective on-line preconcentration and spectrophotometric determination. Anal Chimica Acta. 1995;308(13):425-432.

9. Andrade MGM, Ferreira SLC, Santos BF, Costa ACS. Sequential Determination of Iron And Titanium by Flow-Injection Analysis Talanta. 1992;39(10):1229-1232

10. Lindberg W, Clark GD, Hanna CP, Whitman DA, Christian GD, Ruzicka J. A simple and robust flow-injection analysis method for determination of free acid and metal concentrations in hydrolyzable metal solutions. Anal Chem. 1990;62(8):849-852.
11. Farrukh MA, Siraj N, Aziz-Ur-Rehman, Naqvi II. Comparative study of spectroscopic techniques for the estimation of iron in apple and vegetables. J Saudi Chem Soc. 2010;14(2):209-212.

12. Kuroda R, Nara T, Oguma K. Simultaneous determination of iron(III) and total iron by flow injection analysis using kinetic spectrophotometry with tiron. Analyst. 1988;113:1557-1560.

13. Elmagirbi A, Sulistyarti H, Atikah A. Study of ascorbic acid as iron (III) reducing agent for spectrophotometric iron speciation. J Pure App Chem. Res. 2012;1(1):11-17.

14. Adebayo BK, Ayejuyo S, Okoro HK, Ximba BJ. Spectrophotometric determination of iron (III) in tap water using 8-hydoxyquinoline as a chromogenicreagent. Af.J.Biotech. 2011;10(71):16051-16057. doi: $10.5897 /$ AJB10.1840

15. Gay C, Collins J, Gebicki JM. Determination of Iron in Solutions with the Ferric-Xylenol Orange Complex. Anal Biochem. 1999;273(2):143148

16. Makoto 0. The Spectrophotometric Determination of Uranium with Xylenol Orange. Bull Chem Soc Jap. 1963;36(2):140 -142.

17. Manchanda VK, Tomar BS. Chemical Sciences and Engineering BARC HIGHLIGHTS. 1999; 37-38.

18. Andersen JET. A novel method for the filterless preconcentration of iron. Analyst. 2005;130:385-390.

19. Chandramouleeswaran S, Ramkumar J, Basu M. Insight of Solvent Extraction Process: Reassessment of Trace Level Determinations. Anal Chim Acta. 2016;938(28):123-128. doi: 10.1016/j.aca.2016.08.018 